\title{
Measuring binary fluidization of non-spherical and spherical particles using machine learning aided image processing
}

\author{
Cheng $\mathrm{Li}^{1}$, Xi Gao ${ }^{2}$, Steven Rowan ${ }^{1}$, Bryan Hughes ${ }^{3}$, and William Rogers ${ }^{1}$ \\ ${ }^{1}$ National Energy Technology Laboratory Morgantown \\ ${ }^{2}$ Guangdong Technion-Israel Institute of Technology \\ ${ }^{3}$ National Energy Technology Laboratory
}

August 17, 2021

\begin{abstract}
The binary fluidization of Geldart-D type non-spherical wood particles and spherical LDPE particles was investigated in a laboratory-scale bed. The experiment was performed for varying static bed height, wood particles count, as well as superficial gas velocity. The LDPE velocity field were quantified using Particle Image Velocimetry (PIV). The wood particles orientation and velocity are measured using Particle Tracking Velocimetry (PTV). A machine learning pixel-wise classification model was trained and applied to acquire wood and LDPE particle masks for PIV and PTV processing, respectively. The results show significant differences in the fluidization behavior between LDPE only case and binary fluidization case. The effects of wood particles on the slugging frequency, mean, and variation of bed height, and characteristics of the particle velocities/orientations were quantified and compared. This comprehensive experimental dataset serves as a benchmark for validating numerical models.
\end{abstract}

Measuring Binary Fluidization of Non-spherical and Spherical Particles Using Machine Learning Aided Image Processing

Cheng $\mathrm{Li}^{1 *}$, Xi Gao ${ }^{3 *}$, Steven L. Rowan ${ }^{1,2}$, Bryan Hughes ${ }^{1,2}$, William A. Rogers ${ }^{1}$

1. National Energy Technology Laboratory, Morgantown, WV 26506, USA

2. Leidos Research Support Team, Morgantown, WV 26506, USA

3. Department of Chemical Engineering, Guangdong Technion-Israel Institute of Technology, Shantou, Guangdong 515063, China

*Corresponding author: chengli2.718@gmail.com (C. Li); xi.gao@gtiit.edu.cn (X.Gao),Tel.: +1 304285 0989

\begin{abstract}
The binary fluidization of Geldart-D type non-spherical wood particles and spherical LDPE particles was investigated in a laboratory-scale bed. The experiment was performed for varying static bed height, wood particles count, as well as superficial gas velocity. The LDPE velocity field were quantified using Particle Image Velocimetry (PIV). The wood particles orientation and velocity are measured using Particle Tracking Velocimetry (PTV). A machine learning pixel-wise classification model was trained and applied to acquire wood and LDPE particle masks for PIV and PTV processing, respectively. The results show significant differences in the fluidization behavior between LDPE only case and binary fluidization case. The effects of wood particles on the slugging frequency, mean, and variation of bed height, and characteristics of the
\end{abstract}


particle velocities/orientations were quantified and compared. This comprehensive experimental dataset serves as a benchmark for validating numerical models.

Keywords: Non-spherical particles; Biomass utilization; Image processing; Machine learning; Fluidization

\section{Introduction}

The utilization of both plastic particles and biomass in a fluidized bed system has become increasingly popular and is investigated in many studies due to their great potential for sustainable energy conversion processes such as combustion, gasification, and pyrolysis, to reduce greenhouse gas emissions and abundant supply of the raw material ${ }^{1-3}$. Most of these recycled raw materials are physically processed into granular materials of non-spherical shape. This poses additional complications in establishing accurate predictions of their behavior during applications since the non-spherical particle-particle interaction and particle-fluid interaction behave rather differently compared to spherical particles ${ }^{4}$. Such difference is mainly related to particle orientation and corresponding differences in particle stresses. For example, non-spherical particles with high surface-to-volume ratios can have $20 \%$ - $120 \%$ higher average shear particle stress than that of spheroidal paricles ${ }^{5}$; a free-falling cylinder will align itself with the axis parallel to the flow for moderate Reynolds numbers ${ }^{6}$; non-spherical particles of large aspect ratio are difficult to be fluidized due to interlocking between particles ${ }^{7}$. Spherical particles are usually used to assist the fluidization of non-spherical particles in typical industrial applications ${ }^{8}$. Due to the difference in particle physical properties, such as shape, size, and density, the non-spherical particle can affect the fluidization of spherical particles, such as the transition of fluidization regimes ${ }^{9}$, changes of fluctuation frequency ${ }^{10}$, and so on. Moreover, the non-spherical particles can separate from spherical particles, which leads to segregation. However, segregation is not preferable because non-uniform mixing can significantly decrease the bed performance. Understanding the dynamics of non-spherical particles and the effects of non-spherical particles on binary fluidization is critical for the understanding of fundamental physics and the associated industrial applications.

In situ experimental studies on non-spherical particle dynamics in fluidized beds were seldomly reported in the literature as related experiments to resolve particle-scale information such as position, orientation, velocity, and size are rather costly and complicated. Buist et al. ${ }^{11}$ measured the translational and rotational velocities of cylindrical particles with varying elongation ratios using magnetic particle tracking (MPT) in a cylindrical fluidized bed with $17.4 \mathrm{~cm}$ in diameter. Fotovat et al. ${ }^{12}$ investigated the biomass particle shape factor on the biomass distribution and velocity profiles in a spherical-particles-assisted binary fluidization system using the radioactive particle tracking (RPT) method. Chen et al. ${ }^{13}$ measured the 3D particle position and velocity of a single tagged cylindrical particle over a long period in the binary fluidized bed using X-ray particle tracking velocimetry (XPTV). Vollmari et al. ${ }^{14}$ investigated the distribution and orientation statistics of non-spherical particles in a rectangular bed using the in-house image analysis algorithms. Studying the dynamics of particles through processing images obtained from a high-speed and high-resolution camera is a relatively straightforward method of acquiring quantitative data compared to other complex measurement techniques discussed above.

Efforts have been made to increase the capability of particle scale imaging techniques to higher particle volume fractions by improving the particle detection algorithms or limiting the sample depth. In the processing of the image for non-spherical particle and spherical particle binary fluidization, the main challenge is the segmentation of the non-spherical particles from the spherical particle and background. Segmentation is a process to partition an image into multiple parts or segments. Classical image segmentation methods include histogram thresholding, edge detection based, relaxation, and semantic and syntactic approaches ${ }^{15}$. Each approach has its advantages and limitations. For example, region-based segmentation separates the objects into a different region based on an automatically or manually determined threshold value. It is simple, fast, and performs well when the target object and background have high contrast. However, the accuracy of this method becomes very low when the contrast is low and there is a large overlap. Some studies employing classical image segmentation are available in the literature. Yin et al. ${ }^{16}$ applied an image multilevel thresholding 
approach using the k-means algorithm to identify clusters in a fluidized bed riser. Jiang et al. ${ }^{17}$ employed a particle-mask correlation segmentation approach to detect the particle geometric center in a 2D fluidized bed. In recent years, the machine learning approach demonstrates great promise in the field of particle-scale data extraction from images in multiphase flow research ${ }^{18-24}$. For example, Yevick et al. ${ }^{18}$ measured particle size and positions from analyzing the holographic video microscopy data using machine learning techniques based on support vector machines (SVMs) in real-time on low-power computers. Shao et al. ${ }^{24}$ developed a convolutional neural network (CNN)-based approach for measuring the $3 \mathrm{D}$ particle distributions using digital in-line holography.

The aim of this study is to experimentally investigate the cylindrical particle dynamics and their impact on binary fluidization using image processing and pressure signal analysis. In section 2 , the experiment setups and the methodology of the machine learning-enabled image processing methods are introduced. In section 3 , the results and discussion of pure LDPE sphere fluidization behavior, effects of cylindrical particles mass fraction, superficial gas velocity, and sphere inventory on the cylinder dynamics and fluidization behavior were presented. In section 4 , the results were summarized and further discussion was presented.

\section{Experiments and methodologies}

\subsection{Material characterization}

In the current study, the standard cylindrical hardwood pellets and spherical LDPE particles, both belonging to the Geldart D type, have been used as the test material. The characterization has been conducted following the procedures established in Tucker et al.$^{26}$. Briefly, the particle size and shape statistics of the spherical LDPE have been obtained using a SympaTEC - QICPIC@ particle analyzer which is compliant with the ISO 13322-1/2 Standard. The principle of QICPIC@ operation is based on the analysis of dynamic particle images obtained from backlighting a thin sheet of passing particle samples. Calculation of the minimum superficial gas velocity has been performed.

An in-house small-scale fluidization test has been performed to determine the minimum superficial gas velocity $(U \mathrm{mf})$ of the LDPE particles by determining the pressure drop over the bed as a function of the superficial gas velocity. The resulting $U \mathrm{mf}$ is used to non-dimensionalize the superficial gas velocity for all test cases in the study. All wood pellets have been cut into the same length with aspect ratio, $A R=L / D$ $=2$, where $L$ and $D$ correspond to the length and diameter of the cylindrical wood pellets, respectively. The size of the wood pellet is too large for standard QICPIC@ analysis, thus standard imaging is applied to measure the particle dimensions, to ensure the monodisperse nature of the prepared wood pellets. All related material properties have been summarized in Table 1.

Table 1. Properties of the cylindrical and spherical particles.

\begin{tabular}{lll}
\hline Parameters & Sphere (LDPE) & Cylinder (Biomass pellets) \\
\hline Size $(\mathrm{mm})$, Diameter $-D$, Length $-L$ & $D=3.75$ & $D=6.6, L=13.2$ \\
Particle density $\left(\mathrm{g} / \mathrm{cm}^{3}\right)$ & 0.931 & 1.158 \\
Particle sphericity & 0.929 & 0.832 \\
Minimum fluidization velocity $(\mathrm{m} / \mathrm{s})$ & 0.782 & $\mathrm{~N} / \mathrm{A}$ \\
Group of Geldart's classification & Class D & Class D \\
\hline
\end{tabular}




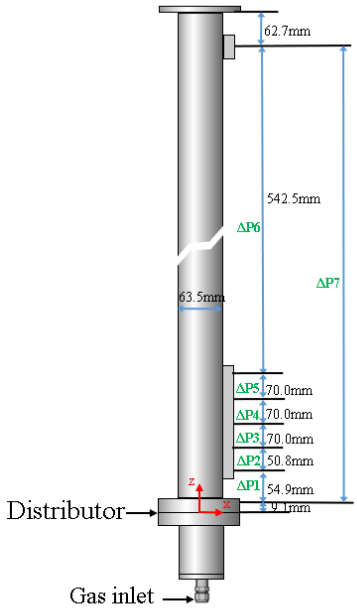

(a)

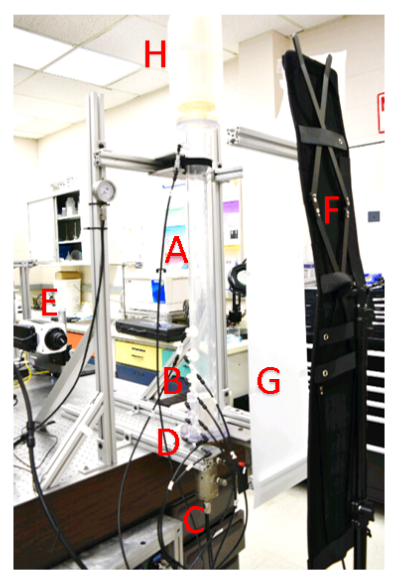

(b)
A - 2.5-inch diameter

fluidized bed

$\mathrm{B}$-Differential pressure

sensors

$\mathrm{C}$ - Gas inlet

$\mathrm{D}$ - Porous distributor

E - Phantom V641 high

speed camera

F - LED light panel

G - Diffuse paper

$\mathrm{H}$ - HEPA filter

Figure 1 . (a) The geometry of the fluidized bed and (b) fluidization and visualization systems.

\subsection{Fluidization and visualization system}

The fluidized bed and the corresponding high-speed visualization setup are shown in Figure 1. The cylindrical fluidized bed contains an optically-accessible acrylic column with an inner diameter of $63.5 \mathrm{~mm}$ and a height of $930.0 \mathrm{~mm}$ (Figure 1a). The coordinate system used in the paper has been indicated in Figure 1a, with the origin at the center of the distributor plate's upper surface, the $x$-axis pointing to the right, and the $z$-axis pointing upward. Also shown in Figure 1a, seven pressure differentials across two different axial locations $(\Delta \mathrm{P} 1-\Delta \mathrm{P} 7)$ have been measured at $100 \mathrm{~Hz}$ using high-frequency pressure transducers with ranges of \pm 1866 $\mathrm{Pa}, \pm 1866 \mathrm{~Pa}, \pm 1866 \mathrm{~Pa}, \pm 1244 \mathrm{~Pa}, \pm 1244 \mathrm{~Pa}, \pm 1244 \mathrm{~Pa}, 0-7465 \mathrm{~Pa}$, respectively. The top of the acrylic column is connected to a HEPA filter, whereas the bottom is connected to a plenum chamber. A distributor plate is placed between the two flanges connecting the plenum and the acrylic column. The plate is made out of sintered metal and is rated at 40 media grade (Mott Corp). In a typical spouted bed facility containing coarse particulate solids, fluid is often injected vertically through a centrally located opening at the base of the bed ${ }^{27}$. Note, such configuration has not been implemented in the current setup. The fluidization gas is supplied into the plenum chamber from a centralized air compressor system at the desired flow rate using an Alicat Scientific mass flow controller, with a range of $0-500$ SLPM and accuracies of $\pm(0.8 \%$ of reading $+0.2 \%$ of full scale). Both the pressure transmitters and the flow controllers are calibrated annually. Due to safety concerns, the absolute pressure of the system is restricted by connecting the plenum chamber to a rupture disk rated at 1.03 bar.

The experimental setup used to visualize the co-fluidization of the mixture is shown in Figure 1b. The fluidized bed is backlit using a rectangular white LED panel. For improving background inhomogeneity, a diffuse paper was placed between the bed and the LED panel. The particle motions are captured using a Phantom v641 CMOS camera equipped with a Nikon@ . 50-mm lens operating at both 100 and 800 frames per second (fps). The physical parameter space of the experiments has been summarized in Table 2, with each case representing a different combination of bed material. Case 1 provides the LDPE particles fluidization case as a benchmark and Case 2 - 4 provides co-fluidization of LDPE particles and wood pellets investigating the effects of static bed height, wood particles fraction on the fluidization behavior of the binary mixing. For each case, the effects of superficial gas velocity had also been investigated. There is a slight variation on mean wood pellets weight, but the variation is within $6 \%$ of the mean wood pellets mass. Also, the wood pellets 
mass after the experiment has also been measured with a negligible amount of mass loss due to attrition.

Table 2. Summary of parameter space.

\begin{tabular}{|c|c|c|c|c|c|}
\hline Case \# & Wood pellets mass $(\mathrm{g})$ & Wood pellets count & LDPE particles mass $(\mathrm{g})$ & Static bed height of LDPE $(\mathrm{cm})$ & Stat \\
\hline 1 & - & - & 149.3 & 7.62 & 1.4 , \\
\hline 2 & 42.7 & 100 & 149.3 & 7.62 & 1. \\
\hline 3 & 22.7 & 50 & 298.6 & 15.24 & 3.0 \\
\hline 4 & 42.7 & 100 & 298.6 & 15.24 & 1.5 , \\
\hline 5 & 85.7 & 200 & 298.6 & 15.24 & 3.0 \\
\hline
\end{tabular}

\subsection{Image processing method}

All the image-based 2D measurements have been performed along the $x-z$ plane shown in Figure 1(a). Note, unless specially mentioned, all imaging data have been acquired during the steady-state of the fluidization at least $5 \mathrm{~min}$ after the start of supplying the gas flow. The lower frame rate data (100 fps) have been used for extracting the expanded bed height temporal evolution matching the acquisition frequency of pressure signals. The detailed image processing algorithms have been reported in Gao et al . ${ }^{7}$ Briefly, the processing steps include, (1) identifying the region of interest and global thresholding using Otsu mehod ${ }^{28},(2)$ filtering $^{2}$ out sticky particles clusters on the wall due to electrostatic forces using morphological and size filters, and (3) estimating the bed height as the maximum height that particle pixel fraction at which height reduces to $5 \%$. The times series are used later for the calculation of the mean and standard deviation of bed height, as well as the bed height spectrum. At least 100 seconds of bed height data has been acquired for statistically robust results. Figure 2 provides a sample convergence test of velocity statistics for the bed height data on mean bed height and standard deviation of bed height as a function of sample time length, denoted as $<H_{\mathrm{b}}>_{t}$ and $\sigma_{H_{b, t}}$. As it shows, both the mean and the standard deviation value fluctuate intensely for $\sim 10$ second and approaches to a constant value as the sample time length increases.

The higher frame rate particle image series at $800 \mathrm{fps}$ have been recorded to acquire the particle velocity and wood particle orientation statistics. The processing steps involve three phases: (a) segmentation of particle types and background, (b) generate particle images containing only one component, (c) perform PIV or PIV on the corresponding image series. During phase (a), owing to the complexity of the backlit images due to illumination and binary particle spatial distribution, segmentation based on global or adaptive local thresholding methods has been proved inadequate for accurately identifying different particle types. Consequently, a machine learning-based pixel-wise classification has been applied, following Arganda-Carreras et al. ${ }^{29}$ The resulting probability maps of classified pixels are then segmented by Otsu's method. Figure 3 provides a sample image series showing the results of the steps during wood particle extraction. As shown in Figure $3(\mathrm{~b})$, the segmentation results based on the machine learning approach successfully classify all pixels into three categories, namely wood particles (red), LDPE particles (green), and background (purple). Afterward, the wood particle masks are generated (Figure 3c,d), and the geometric parameters including, particle center, width/length, aspect ratio, and orientation are measured (Figure 3e). Due to the inherent limitation in $2 \mathrm{D}$ imaging, the 3D orientation of the particle relative to the bed central axis is not readily measured. To eliminate the limitation, only particles aligning almost parallel to the imaging plane ( $\mathrm{x}-\mathrm{z}$ plane, see Figure 1) are sampled as statistics. This is implemented by filtering out all particles having lengths less than $90 \%$ of the length. The detected wood particles videos are provided as supplemental information. Based on the particle location, wood particles are tracked undergoes particle tracking velocimetry (PTV) to provide Lagrangian particle tracks of the wood pellets, using an algorithm developed previously by Ouellette et al. ${ }^{30}$. Only tracks with lengths larger than 5 frames have been used. Next, the wood pellets are masked out in the original image. The Eulerian velocity fields of the LDPE particles were then computed using a particle image velocimetry (PIV) open software PIVlab ${ }^{31}$, with a final interrogation windows size of $64 \times 64$ pixels with $50 \%$ overlap. Figure 4 shows the sample images illustrating the PIV analysis. Figure 4 a shows a raw 
image with LDPE particles with the inserts showing the particle image of the $64 \times 64$ pixels interrogation window. The LDPE particles mask (Figure $4 \mathrm{~b}$ ) generated using the abovementioned machine-learning algorithm was used for determining the LDPE particle pixel percentage within the interrogation window. Only interrogation windows with LDPE particle pixel percentages larger than $10 \%$ are used for providing velocity values of the LDPE particles as shown in Figure 4c.

\section{Hosted file}

image2.emf available at https://authorea.com/users/430819/articles/534349-measuring-binaryfluidization-of-non-spherical-and-spherical-particles-using-machine-learning-aidedimage-processing

Figure 2. Sample convergence test of the mean, $\left\langle H{ }_{\mathrm{b}}\right\rangle_{t}$ (solid blue line) and standard deviation, $\sigma_{H_{b, t}}$ (orange dashed line) of expanded bed height time series.
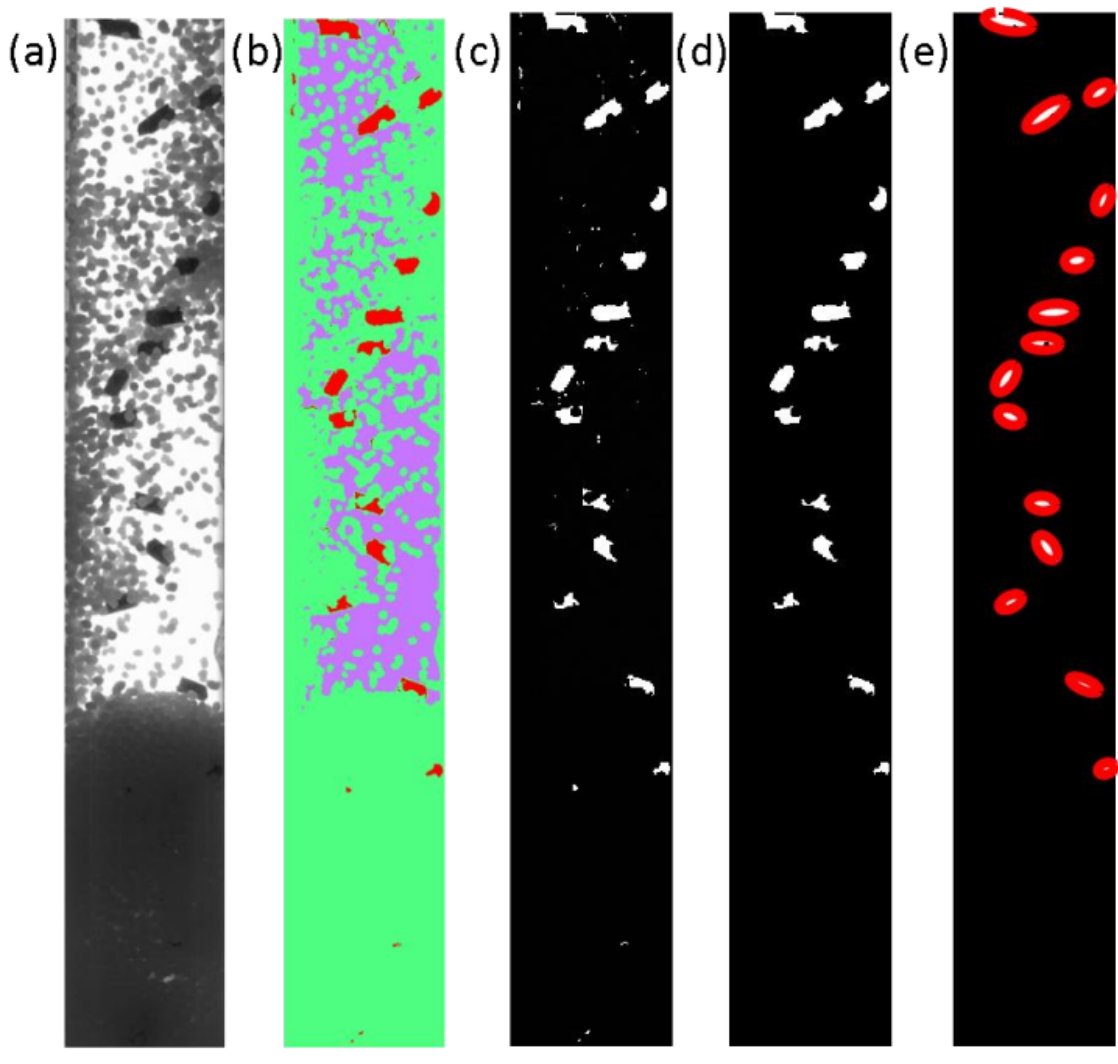

Figure 3 . Segmentation of wood particles. (a) raw particle image, (b) machine learning segmentation results, (c) segmented wood particles, (d) segmented wood particles after noise filtering, and (e) detected wood particles. 

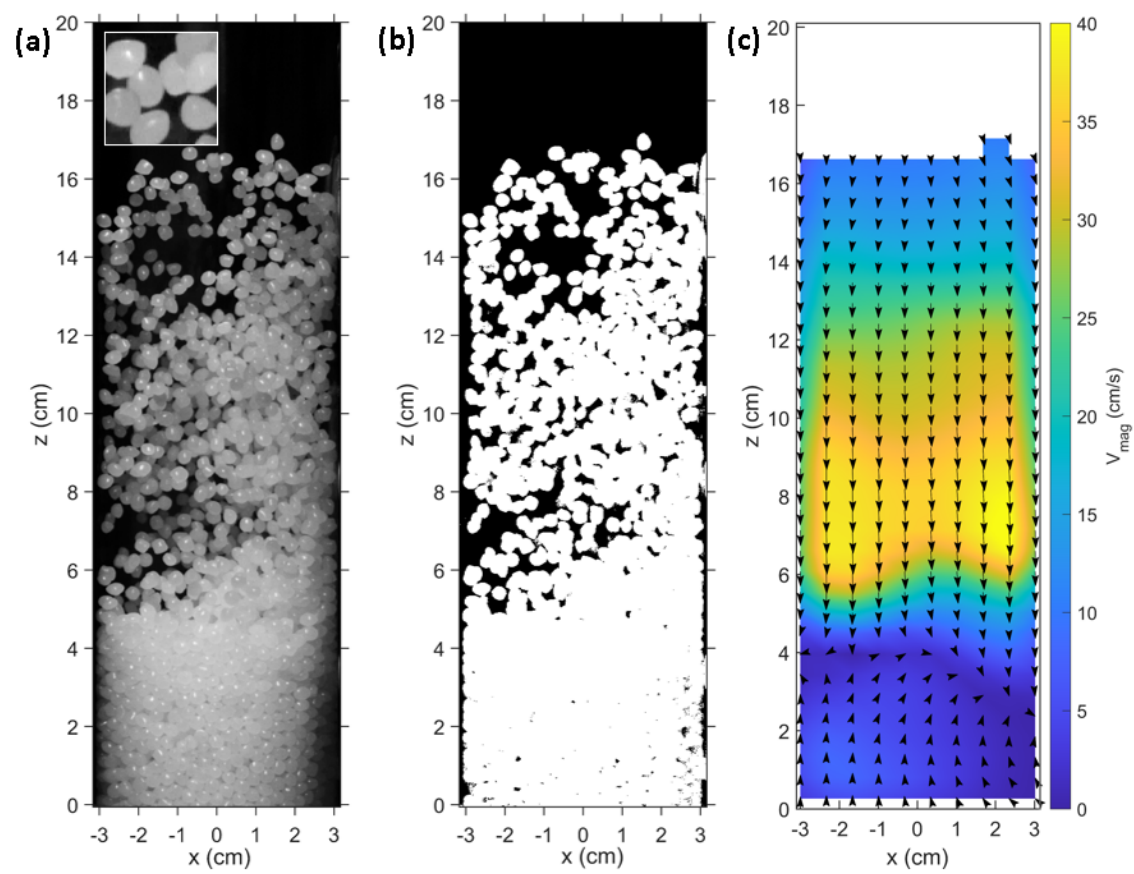

Figure 4 . LDPE particle velocity field extraction using PIV analysis. (a) raw LDPE particle image, (b) LDPE particle masks, (c) particle velocity field.

\section{Results and discussion}

\subsection{LDPE Sphere fluidization}

As a benchmark, case 1 of the study investigates the fluidization behavior of LDPE particles with superficial gas velocity from $1.4 \mathrm{U} \mathrm{mf}$ to $3.4 \mathrm{U}$ m. Figure 5 compares the fluidization behavior of LDPE spherical particles with varying superficial gas velocity. The image frame with the maximum expanded bed height has been chosen for each superficial gas velocity for demonstration purposes (Figure 5a-d). At the lower superficial gas velocity $(1.4 \mathrm{U} \mathrm{mf}$ and $2.0 \mathrm{U} \mathrm{mf})$, slug flows are formed due to the relatively narrow bed configuration, with the maximum expanded bed height increases significantly due to the increase of superficial gas velocity from $1.4 \mathrm{U} \mathrm{mf}$ and $2.0 \mathrm{U} \mathrm{mf}$. Markedly, such a trend ceases with the further increase of superficial gas velocity. At $3.0 \mathrm{U} \mathrm{mf}$ and $3.4 \mathrm{U}$ mf the maximum expanded bed height drops significantly compared to 2.0 $U \mathrm{mf}$ case, and two regions with visually different particle void fraction are formed. Generally similar to a spouted bed, the loosely packed fountain region is at the top of the bed while a densely packed region forms at the bottom. To quantify the overall fountain region length, the 500 frames are overlaid to generate the time-averaged bed images at these fluidization velocities. The two regions are distinct due to the grayscale value and the fountain height is measured to be $3.36 \mathrm{~cm}$ and $5.02 \mathrm{~cm}$ at a superficial gas velocity of $3.0 \mathrm{U}$ $\mathrm{mf}$ and $3.4 \mathrm{U} \mathrm{mf}$, respectively. The current transition from slugging to spouting is similar to the behavior observed in some of the so-called spout-fluid beds using a central jet together with an auxiliary flow through a porous or perforated distributor plate at certain flow conditions ${ }^{33,34}$.

The velocity field for $2 \mathrm{U}_{\mathrm{mf}}$ case is shown in Figure 6 . The color encodes the velocity magnitude, $\mathrm{V}_{\mathrm{mag}}$ of the particles. Due to the narrow bed of solids, at $\mathrm{t}=0 \mathrm{~s}$, the bubbles formed at the distributor plate grow into a flat slug which is clearly identified by the almost uniform upward velocity region of around $20 \mathrm{~cm} / \mathrm{s}$ with a size of around $5 \mathrm{~cm}$ in length. Above the slugs, the particles from previously disintegrated slugs fall onto 
the slug and have a velocity close to zero. Below the slug, the particles have relatively low velocities and separate from the slug. At $\mathrm{t}=0.125 \mathrm{~s}$, the slug continues to move upwards, and in the meanwhile accelerate particles from previous slugs. At the bottom of the slug, the particles that cannot stick to the slug start to replenish by raining solids. The size of the slug remains almost unchanged. At $t=0.313 \mathrm{~s}$, a large portion of the slug's particles starts to fall which spans a length of around $10 \mathrm{~cm}$, whereas the rest of the particles continue to rise and decelerate as shown in the velocity map. At $\mathrm{t}=0.556 \mathrm{~s}$, all the particles in the slug start to fall and reach as high as $56 \mathrm{~cm} / \mathrm{s}$. The particles at bottom of the bed start to form another slug with a small upward velocity. At $\mathrm{t}=0.763 \mathrm{~s}$, the particles continue to fall while another slug continues to form. In general, the dynamics of the LDPE particles at $2 \mathrm{U}_{\mathrm{mf}}$ resembles the typical Geldart type D particles behavior, even though no complete void space or multiple slugs is formed presumably due to the relative shallow bed configuration. The rising rate of the bubble or the slug can be estimated to be around 35 $\mathrm{cm} / \mathrm{s}$ by tracking the almost zero velocity band below the slug. This result is in good agreement with the correlation results proposed by Stewart and Davidson ${ }^{35}$,

$U_{\mathrm{b}}=0.35\left(g D_{\mathrm{t}}\right)^{0.5}$

where $D_{\mathrm{t}}$ is the diameter of the bed, and $g$ is the gravitational constant, which results in a value of $28 \mathrm{~cm} / \mathrm{s}$.
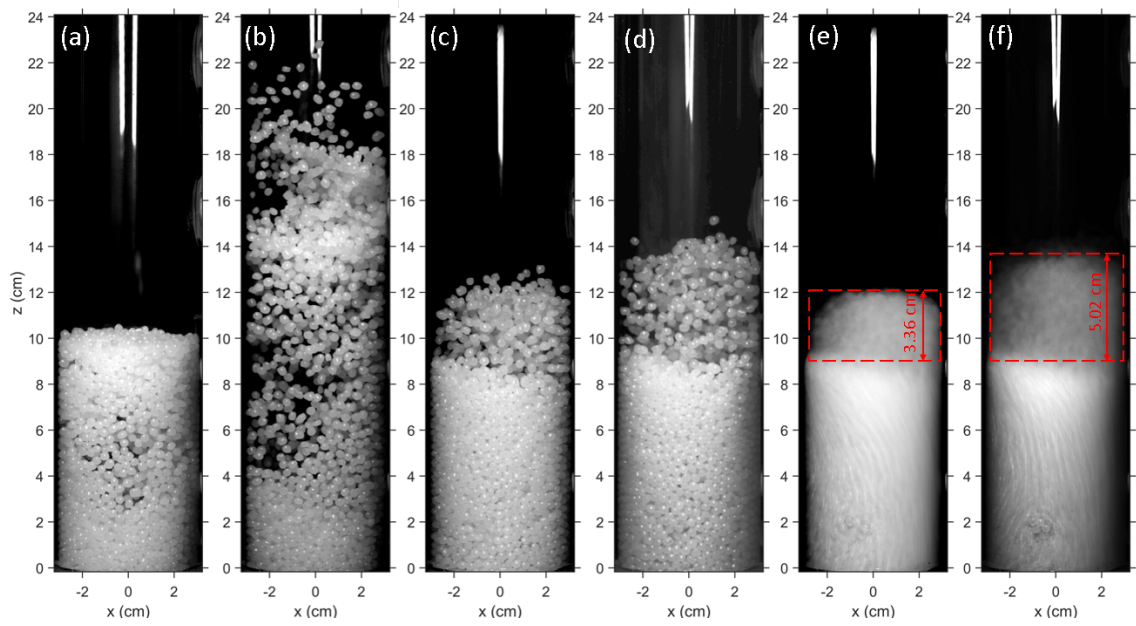

Figure 5. Sample images of spherical LDPE particles fluidization with a superficial gas velocity of (a) $1.4 \mathrm{U}_{\mathrm{mf}}$, (b) $2.0 \mathrm{U}_{\mathrm{mf}}$, (c) $3.0 \mathrm{U}_{\mathrm{mf}}$, (d) $3.4 \mathrm{U}_{\mathrm{mf}}$, and overlay of 500 images of (e) $3.0 \mathrm{U}_{\mathrm{mf}}$ and (f) $3.4 \mathrm{U}_{\mathrm{mf}}$ cases with the red dashed rectangle showing the region of the particle fountain. 

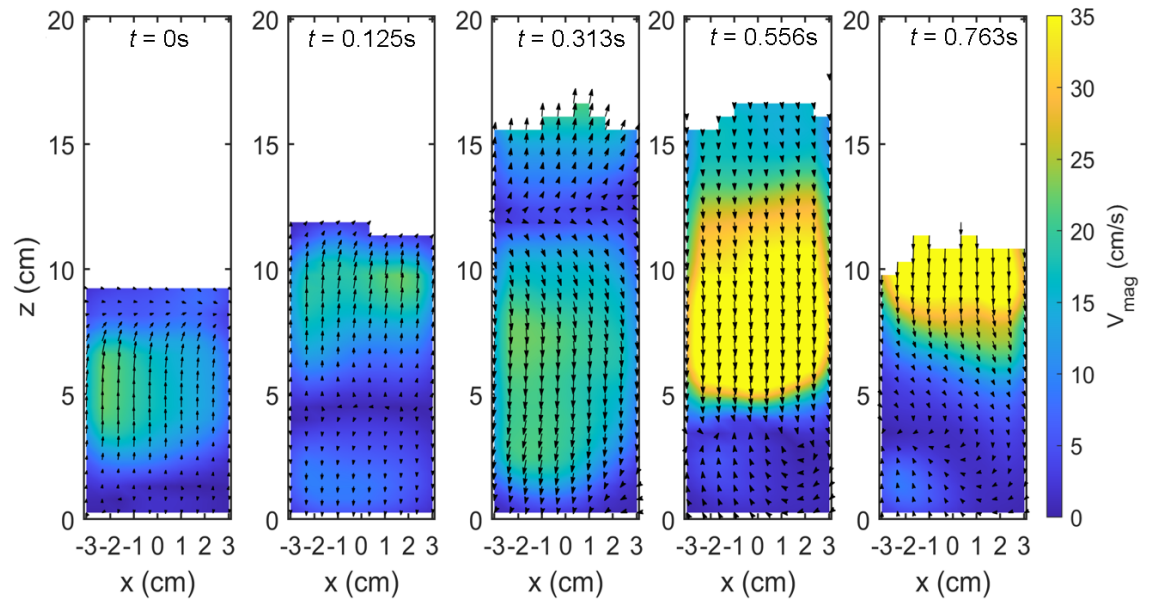

Figure 6. LDPE particle velocity field over time in slugging regime $\left(2.0 \mathrm{U}_{\mathrm{mf}}\right)$
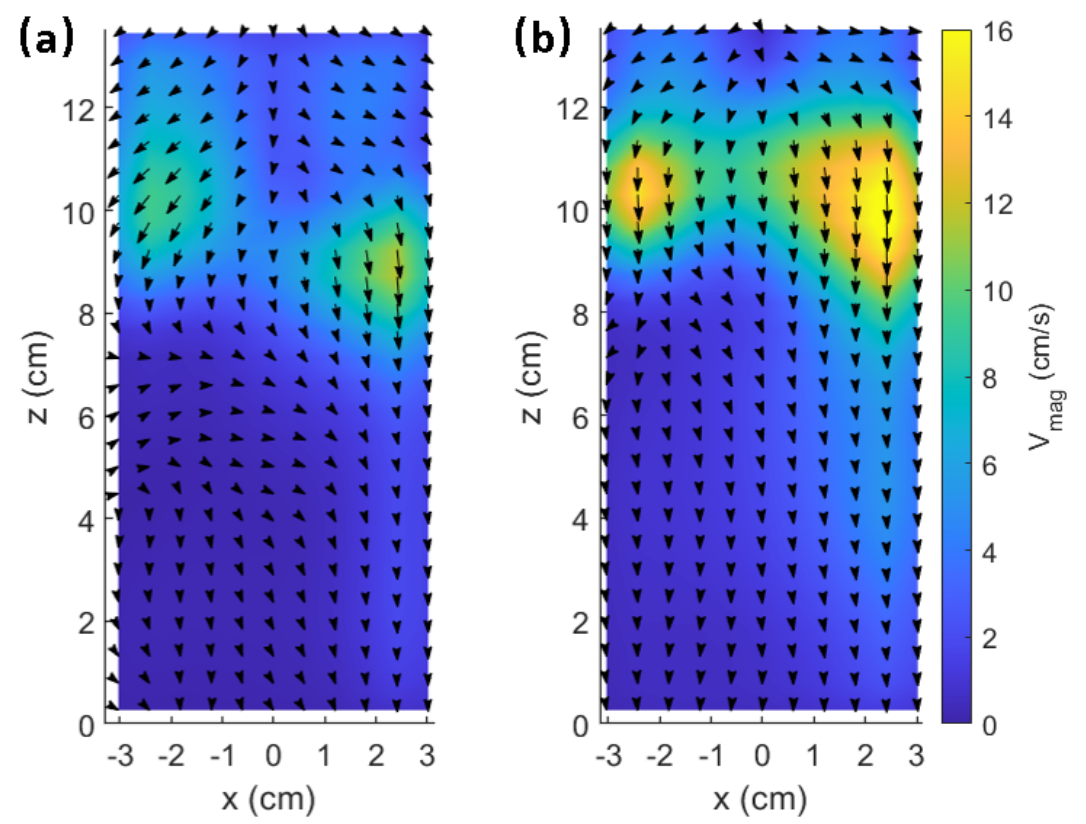

Figure 7. Sample instantaneous LDPE velocity in spouting regime (a) $3.0 \mathrm{U}_{\mathrm{mf}}$ (b) $3.4 \mathrm{U}_{\mathrm{mf}}$.

The sample instantaneous velocity field for both $3 \mathrm{U}_{\mathrm{mf}}$ and $3.4 \mathrm{U}_{\mathrm{mf}}$ cases are shown in Figure 6 . Note, the flow is highly three-dimensional, so the velocity field provided here represents only the particle velocity near the bed wall close to the camera. As it shows, for both cases, the fountain regions at top of the bed have significantly higher velocity magnitude than the rest of the bed. With the increase of gas velocity, the peak velocity also increases. However, the maximum velocity magnitude for both $3.0 \mathrm{U}_{\mathrm{mf}}$ and $3.4 \mathrm{U}_{\mathrm{mf}}$ cases is significantly lower than that for the slugging bed at $2 \mathrm{U}_{\mathrm{mf}}$. Moreover, there seems to be an asymmetry between the fountain region causing the preferential downward flow at the right side of the bed. This may be due to the disturbance of flows introduced during the distributor installation. The bed particle velocity at the 
fountain region has been measured previously in the spout bed ${ }^{36-38}$. He et al. ${ }^{36}$ conducted a comprehensive measurement of profiles of vertical particle velocity in the $1.41 \mathrm{~mm}$ glass beads $\left(U_{\mathrm{mf}}=0.54 \mathrm{~m} / \mathrm{s}\right)$ spouted bed using fiber optics probes. The maximum downward velocity $\mathrm{v}_{\max }$ to $\mathrm{U}_{\mathrm{mf}}$ ratio is measured to be around 2.8 with a central jet velocity of $1.3 \mathrm{U}_{\mathrm{mf}}$. In the current configuration, the corresponding ratio is only around 0.15 and 0.20 for $3.0 \mathrm{U}_{\mathrm{mf}}$ and $3.4 \mathrm{U}_{\mathrm{mf}}$ respectively. The significant decrease in the maximum downward velocity in the fountain region is possibly due to that the current setup does not have the central jet configuration used in the previous study.

The mean and fluctuation of the expanded bed height for all four fluidization velocities are shown in Figure 8. As expected, the bed height fluctuates overtime for all fluidization velocities as gas flow facilitates the formation of moving slugs/fountains. However, the resulting fluctuation intensity and the mean bed height differ. Both the mean and the fluctuation of the bed height increases from $1.4 \mathrm{U}_{\mathrm{mf}}$ to $2.0 \mathrm{U}_{\mathrm{mf}}$. With the increase of superficial gas velocity from $2 \mathrm{U}_{\mathrm{mf}}$ to $3 \mathrm{U}_{\mathrm{mf}}$, both the mean and the fluctuation of bed height decreased significantly due to the onset of spout at $3 \mathrm{U}_{\mathrm{mf}}$. With a further increase of superficial gas velocity to $3.4 \mathrm{Umf}$, the mean bed height increases slightly, whereas the fluctuation of bed height decreases indicating a more stable spouted bed.

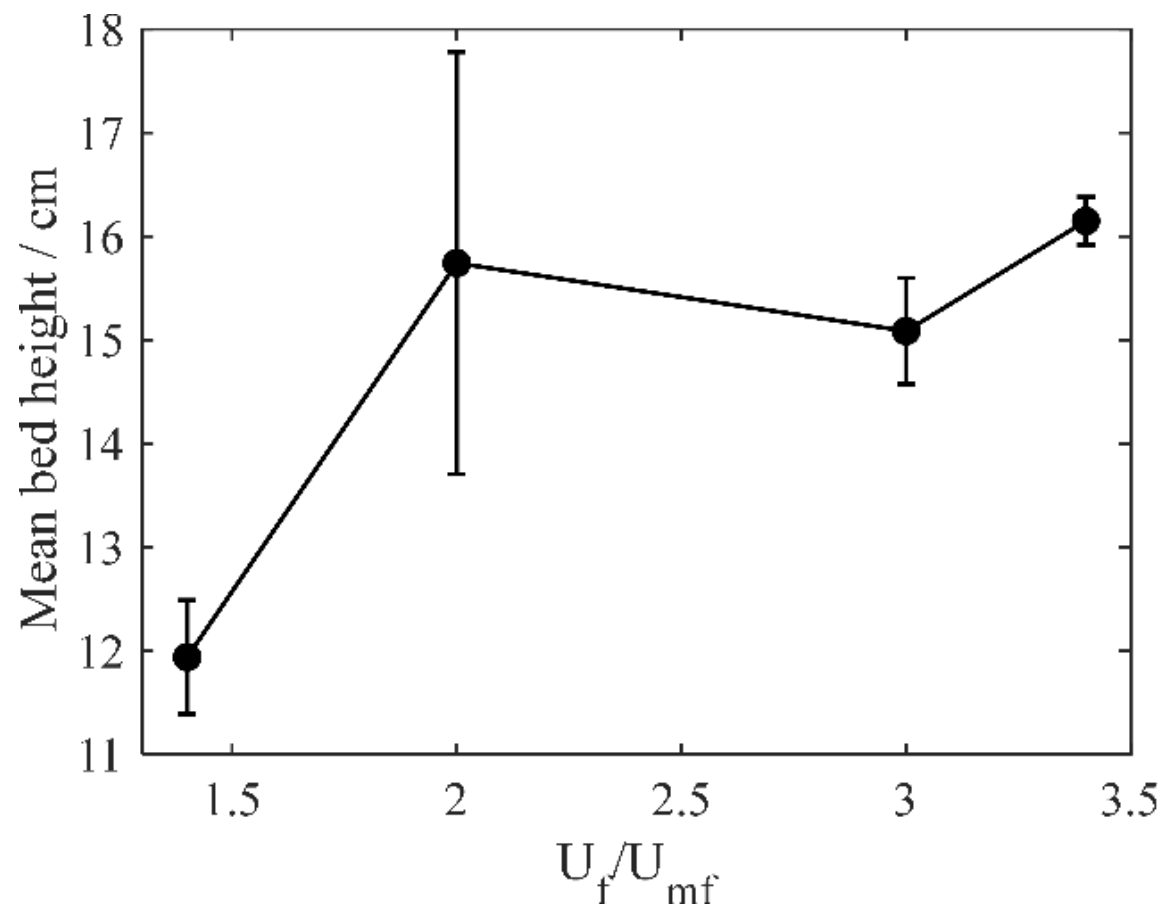

Figure 8 . The mean and standard deviation of the expanded bed height with varying fluidization gas velocity.

\subsection{Effect of wood pellets on the fluidization behavior}

In this section, fluidization with the addition of 100 wood pellets in the bed relative to case 1 was investigated and compared to the baseline. Note, the static bed height provided in Table 2 corresponds to the LDPE particle bed height for all cases. With the addition of 100 wood pellets, the actual bed is around $2.5 \mathrm{~cm}$ higher than the LDPE only case, yet is referred to using the LDPE bed height. Figure 9 shows the snapshots of the binary fluidization of wood and LDPE particles with superficial gas velocity ranging from $1.5-3.7$ $\mathrm{U}_{\mathrm{mf}}$. At $1.5 \mathrm{U}_{\mathrm{mf}}$ (Figure 9a), the particle mixture remain segregated, with wood particles sitting at the bottom of the bed due to its larger density. At $2.0 \mathrm{U}_{\mathrm{mf}}$ (Figure $9 \mathrm{~b}$ ), particle mixture begins to mix, even 
though at higher elevations only LDPE particles are observed. Different from the LDPE only case that forms spouts with the further increase of superficial gas velocity to $3.0 \mathrm{U}_{\mathrm{mf}}$, at $3.0 \mathrm{U}_{\mathrm{mf}}$ and $3.7 \mathrm{U}_{\mathrm{mf}}$, the bed stays in the slugging region with wood particles well mixed with the LDPE particles.

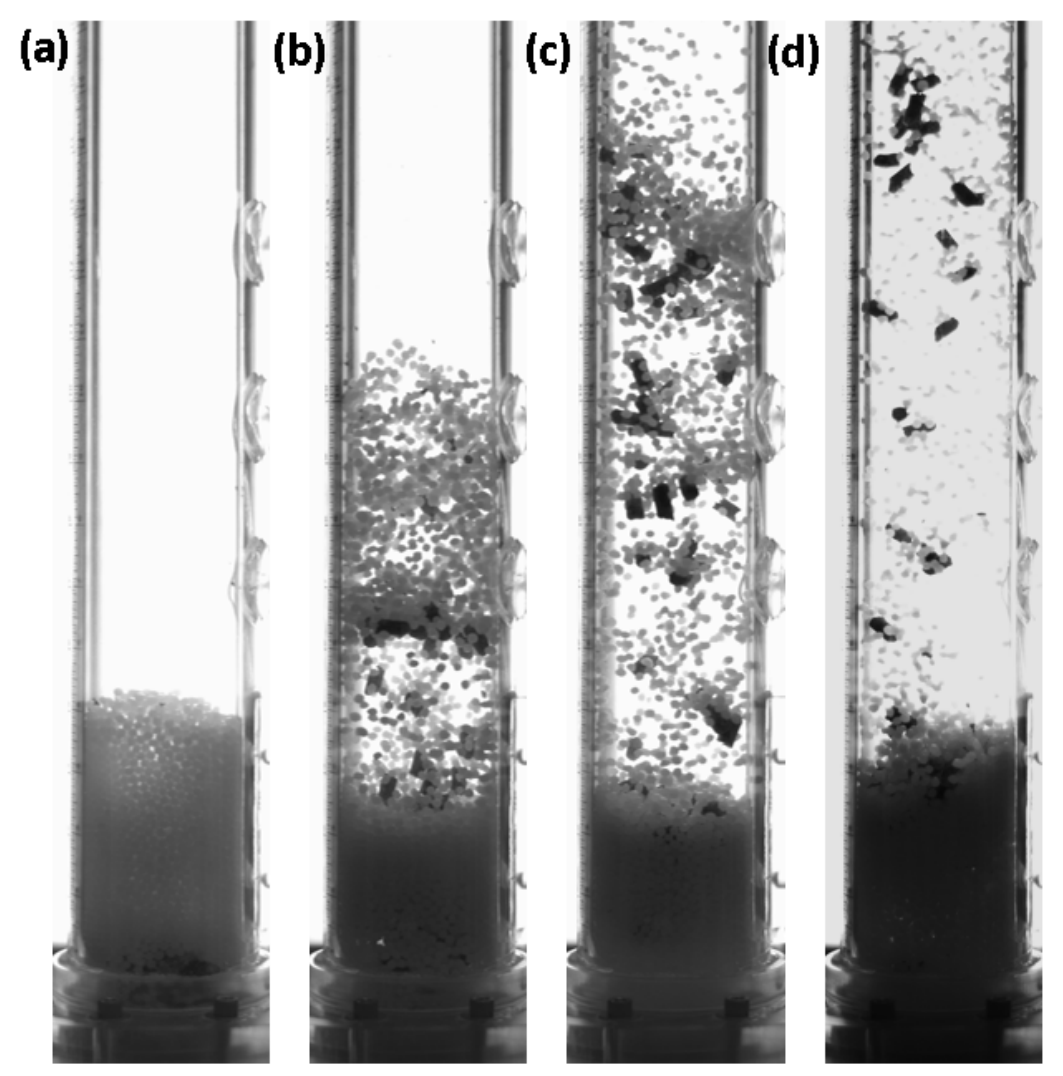

Figure 9 . Sample images of spherical LDPE particles fluidization and 100 cylindrical wood pellets with a superficial gas velocity of (a) $1.5 \mathrm{U}_{\mathrm{mf}}$, (b) $2.0 \mathrm{U}_{\mathrm{mf}}$, (c) $3.0 \mathrm{U}_{\mathrm{mf}}$, (d) $3.7 \mathrm{U}_{\mathrm{mf}}$.

Figure 10 and 11 shows the temporal evolution of the LDPE particle velocity field at $2.0 \mathrm{U}_{\mathrm{mf}}$ and 3.0 $\mathrm{U}_{\mathrm{mf}}$ respectively. The general slugging behavior resembles that observed for the LDPE only case. The process generally involves (1) the slug formation and rising at an almost constant speed (Figure 10: $t=0$ $0.313 \mathrm{~s}$, and Figure 11: $\mathrm{t}=0-0.213 \mathrm{~s}$ ) and (2) disintegration of the slug by raining down the particles (Figure 10: $\mathrm{t}=0.313-0.763 \mathrm{~s}$, and Figure 11: $\mathrm{t}=0.213-0.606 \mathrm{~s})$. However, the characteristic size and velocity of these processes differ significantly between each case. For example, at $2 \mathrm{U}_{\mathrm{mf}}$, both LDPE only case and binary mixture case form a slug of around $5 \mathrm{~cm}$. At $3 \mathrm{U}_{\mathrm{mf}}$ the slug length of the binary mixture case increases to $14 \mathrm{~cm}$. As for the slug rising speed, at $2 \mathrm{U}_{\mathrm{mf}}$, the rising speed is $35 \mathrm{~cm} / \mathrm{s}$ and $44 \mathrm{~cm} / \mathrm{s}$ for LDPE only case and the binary fluidization case respectively. At $3 \mathrm{U}_{\mathrm{mf}}$, the rising speed of the slug increases to $101 \mathrm{~cm} / \mathrm{s}$. Similarly, at $2 \mathrm{U}_{\mathrm{mf}}$, the maximum falling speed is $56 \mathrm{~cm} / \mathrm{s}$ for the LDPE only case, and increases to $119 \mathrm{~cm} / \mathrm{s}$ for the binary fluidization case. At $3 \mathrm{U}_{\mathrm{mf}}$, it reaches as high as $224 \mathrm{~cm} / \mathrm{s}$.

To compare the general trends of fluidization with and without non-spherical wood pellets, the spatially averaged vertical velocity is calculated using all available velocity vectors in the field of view, denoted as $<v>_{\text {FOV }}$. The comparison of the temporal evolution of $\left\langle v>_{\text {FOV }}\right.$ for LDPE only and binary fluidization case at both $2 \mathrm{U}_{\mathrm{mf}}$ and $3 \mathrm{U}_{\mathrm{mf}}$ is shown in Figure 12. As expected, the LDPE only case at $3 \mathrm{U}_{\mathrm{mf}}$ remains almost constant due to the transition from the slugging to the spouting regime. Except for the LDPE only 
case at $3 \mathrm{U}_{\mathrm{mf}}$, the $\left\langle v>_{\mathrm{FOV}}\right.$ varies quasi periodically with varying amplitude and frequency. To quantify the amplitude and frequency, the local troughs and peaks are detected for the whole duration of the available dataset. The corresponding amplitude and period are measured and the mean values are calculated across all detected local troughs and peaks. At $2 \mathrm{U}_{\mathrm{mf}}$, the mean amplitude of the $\langle v\rangle_{\text {FOv signal increases significantly }}$ with the addition of wood pellets from $29.4 \mathrm{~cm} / \mathrm{s}$ to $58.7 \mathrm{~cm} / \mathrm{s}$. At $3 \mathrm{U}_{\mathrm{mf}}$ the mean amplitude for the binary fluidization case increases to $123.0 \mathrm{~cm} / \mathrm{s}$. The mean frequency of the signal is $1.72 \mathrm{~Hz}$ for LDPE only case at $2 \mathrm{U}_{\mathrm{mf}}$ and increases to $2.37 \mathrm{~Hz}$ with the addition of wood pellets at the same superficial gas velocity. At $3 \mathrm{Umf}$, the mean frequency decreases significantly to $1.54 \mathrm{~Hz}$, presumably due to the longer traveling range of the slugs in the bed.
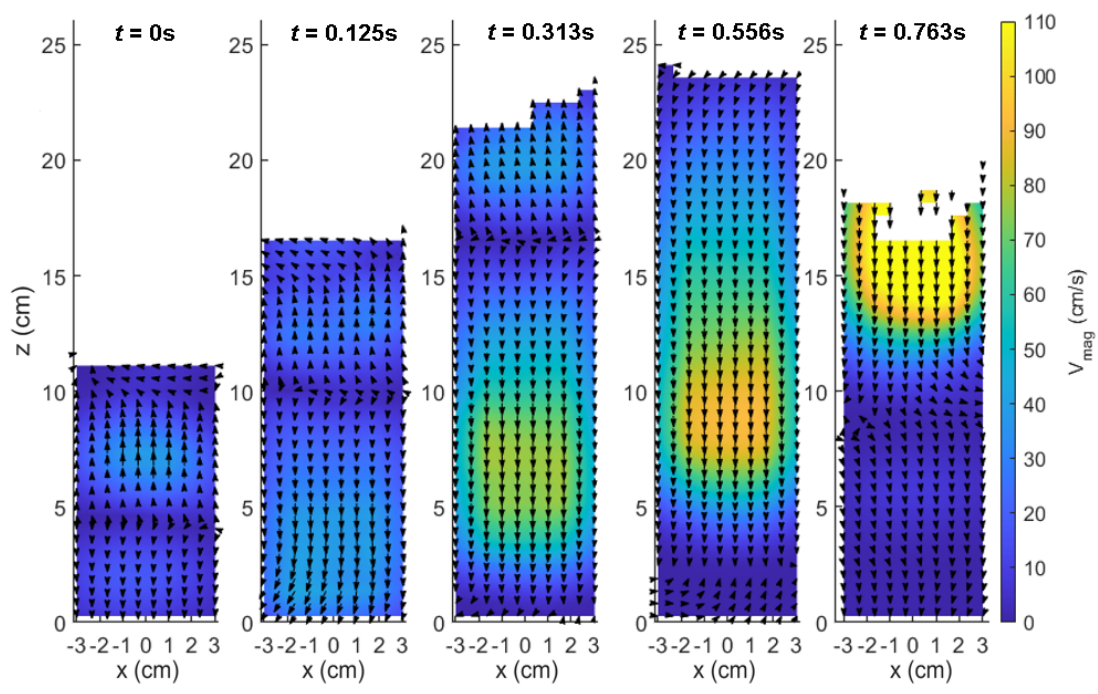

Figure 10 . The temporal evolution of LDPE particle velocity field overtime at $2.0 \mathrm{U}_{\mathrm{mf}}$. 


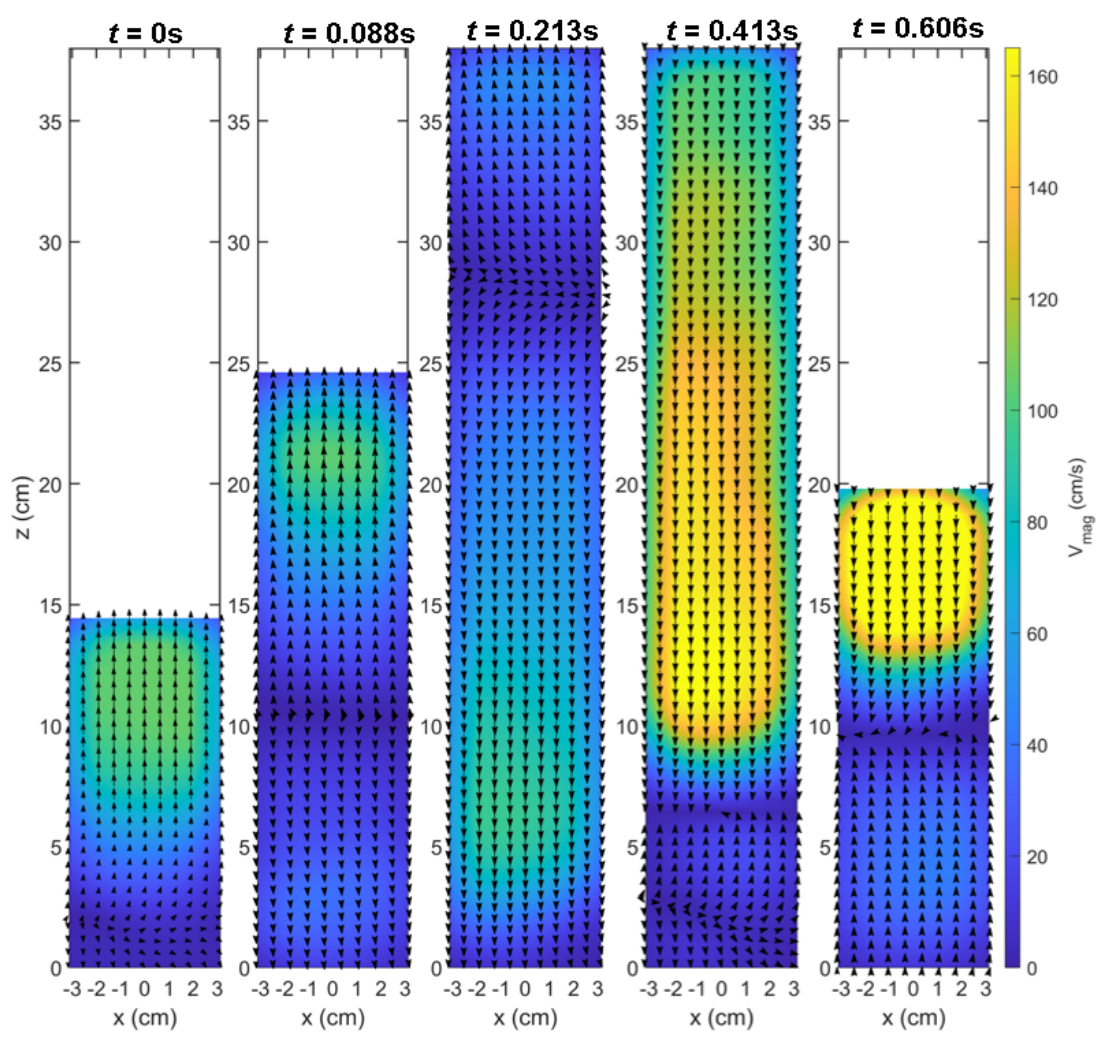

Figure 11. The temporal evolution of LDPE particle velocity field overtime at $3.0 \mathrm{U}$ mf

\section{Hosted file}

image12.emf available at https://authorea.com/users/430819/articles/534349-measuring-binaryfluidization-of-non-spherical-and-spherical-particles-using-machine-learning-aidedimage-processing

Figure 12. The comparison of the temporal evolution of spatially averaged LDPE particle velocity for case 1 and case 2 at $2 \mathrm{U}_{\mathrm{mf}}$ and $3 \mathrm{U}_{\mathrm{mf}}$.

\subsection{Effect of the static bed height and wood particle counts on the fluidization behavior}

\section{Hosted file}

image13.emf available at https://authorea.com/users/430819/articles/534349-measuring-binaryfluidization-of-non-spherical-and-spherical-particles-using-machine-learning-aidedimage-processing

Figure 13. The nondimensionalized mean and standard deviation of bed height for LDPE and 100 wood pellets binary mixture with varying initial bed height.

Figure 13 shows the effects of initial bed height on the mean and standard deviation of bed height for LDPE and 100 wood pellets binary mixture. After non-dimensionalizing the mean bed height and standard deviation with the initial bed height $\left(\mathrm{H}_{\mathrm{b} 0}\right)$, both curves are well collapsed on a straight line from $1.5 \mathrm{U}_{\mathrm{mf}}$ to $3 \mathrm{U}_{\mathrm{mf}}$ range, with both the nondimensionalized bed height and standard deviation increase with the 
increase of the superficial gas velocity. The experimental data above $3 \mathrm{U}_{\mathrm{mf}}$ is only available for shallower bed cases due to pressure constraints for the facility, which shows that the mean bed height remains almost constant at $3.7 \mathrm{U}_{\mathrm{mf}}$. This trend is consistent with the pressure drop data reported in Li et al. ${ }^{25}$. Figure 14 shows the effects of initial bed height on the temporal evolution of the spatially averaged vertical velocity. In presenting the data, $\langle\mathrm{v}\rangle_{\text {FOV }}$ is non-dimensionalized using the characteristic velocity $(\mathrm{g} \Delta \mathrm{H})^{1 / 2}$, where $\Delta \mathrm{H}=\mathrm{H}_{\mathrm{b}}-\mathrm{H}_{\mathrm{b} 0}$, is the difference between expanded and initial bed height. The time is non-dimensionalized using the characteristic time for the particle to travel $\Delta \mathrm{H}$, i.e. $\tau=(2 \Delta \mathrm{H} / \mathrm{g})^{1 / 2}$. The non-dimensionalized periods are at $\sim 3$. Specifically, it is 3.25 at $2 \mathrm{U}_{\mathrm{mf}}$ and 2.66 at $3 \mathrm{U}_{\mathrm{mf}}$ for the shallower bed and 2.89 at $2 \mathrm{U}_{\mathrm{mf}}$ and 3.10 at $3 \mathrm{U}_{\mathrm{mf}}$ for the deeper bed. The non-dimensionalized amplitude is 1.71 and 1.36 at $2 \mathrm{U}_{\mathrm{mf}}$ and $3 \mathrm{umf}$ respectively for the deep bed. The value decreases to 0.78 and 0.92 at $2 \mathrm{U}_{\mathrm{mf}}$ and $3 \mathrm{U}_{\mathrm{mf}}$ respectively for the shallow bed. In general, the nondimensionalization works reasonably well for each static bed height with varying superficial gas velocity, yet works less satisfactory when static bed height is changed. This is possibly due to the internal flow structure change due to the increase of the static bed height.

\section{Hosted file}

image14.emf available at https://authorea.com/users/430819/articles/534349-measuring-binaryfluidization-of-non-spherical-and-spherical-particles-using-machine-learning-aidedimage-processing

Figure 14. Comparison of nondimensionalized spatially averaged LDPE particle velocity over time with varying static bed height and fluidization gas velocity.

\section{Hosted file}

image15.emf available at https://authorea.com/users/430819/articles/534349-measuring-binaryfluidization-of-non-spherical-and-spherical-particles-using-machine-learning-aidedimage-processing

Figure 15. The distribution of the orientation for the wood particles with varying bed height and superficial gas velocity.

\section{Hosted file}

image16.emf available at https://authorea.com/users/430819/articles/534349-measuring-binaryfluidization-of-non-spherical-and-spherical-particles-using-machine-learning-aidedimage-processing

Figure 16. The distribution of the orientation of wood particles at $3 \mathrm{U}_{\mathrm{mf}}, \mathrm{H}_{\mathrm{b} 0}=15.24 \mathrm{~cm}$, and varying wood particle counts.

Figure 15 shows the probability density function (PDF) of wood particle orientation in the fluidized bed with both shallow $\left(\mathrm{H}_{\mathrm{b} 0}=7.62 \mathrm{~cm}\right)$ and deep bed $\left(\mathrm{H}_{\mathrm{b} 0}=15.24 \mathrm{~cm}\right)$ configurations and a superficial gas velocity of $2 \mathrm{U}_{\mathrm{mf}}$ and $3 \mathrm{U}_{\mathrm{mf}}$. The orientation is quantified using the angle between the detected major axis of the wood particle and $z$-axis of the bed, with ranges from $0^{\circ}$ (vertical) to $90^{\circ}$ (horizontal). Currently, the measurements are inherently two-dimensional over the x-z plane which is incapable of measuring accurately all wood particle's orientation relative to z-axis. As an alternative, to minimize the measurement error due to the three-dimensional projection, the orientation statistics samples only particles with an aspect ratio larger than 1.8. Namely, the particles that lie almost in the x-z plane are conditionally sampled in the orientation statistics. As the distribution shows, for all cases, the distribution has a dip around $45^{\circ}$ and slightly favors both the vertical and horizontal orientation, respectively. This presumably corresponds to the wood particle fluidization in the dilute and dense LDPE particle regions of the bed. Namely, the wood particle tends to be horizontal when reaching the dense LDPE particle pool while staying vertical when falling after the slug disintegrates. These results are in qualitative agreement with previous experimental results by Vollmari et al. $2016^{14}$ with a comparable superficial gas velocity range. For example, for the long cylindrical wood particle case $(14 \mathrm{~mm} \times 4 \mathrm{~mm}$ wood cylindrical particle) in the study, the superficial gas velocity varies from 
around 1.6 to $2.4 \mathrm{U}_{\mathrm{mf}}$, all the orientation statistics show local peaks for both vertical and horizontal particle orientation. Even though the experiment was conducted using a single non-spherical particle type. Figure 16 shows the orientation distribution of the wood particles at a fixed superficial gas velocity $\left(3 \mathrm{U}_{\mathrm{mf}}\right)$ and a deep bed configuration with varying wood particle counts from $50-200$. The results show similar trends as described above without significant variation between the tested cases.

Apart from the orientation statistics of the wood particles, the velocity has also been acquired based on the tracked wood particles. Figure 17 shows both the horizontal and vertical velocity of wood particles centroid. As expected, for horizontal velocity (Figure 17a), the distributions are well presented by a normal distribution with a mean of around zero for all cases. The standard deviation increases slightly with the increase of superficial gas velocity from $2 \mathrm{U}_{\mathrm{mf}}$ to $3 \mathrm{U}_{\mathrm{mf}}$, and decreases with the increase of the static bed height from $7.62 \mathrm{~cm}$ to $15.24 \mathrm{~cm}$. For the vertical velocity (Figure 17b), the distributions are non-gaussian and spread more widely compared to the horizontal velocity from around $-3 \mathrm{~m} / \mathrm{s}$ to $3 \mathrm{~m} / \mathrm{s}$. As the superficial gas velocity increases with a fixed static bed height, the standard deviation also increases. For the $\mathrm{H}_{\mathrm{b} 0}=15.24 \mathrm{~cm}$ case, the distribution becomes bimodal. With the increase of the bed height, the distribution shifts to more negative values possibly due to the increase of mean bed height (c.f. Figure 13) and thus an increase in the downward wood particle velocity. Furthermore, the effects of wood particle counts on the wood particle velocity are shown in Figure 18. Similarly, the distributions remain a normal distribution with zero mean for all cases. The standard deviation increases slightly with the increase of wood particle counts. For the vertical velocity distribution, all three cases are bimodal. The first mode is at around zero whereas the second mode is centered at $-1.5 \mathrm{~m} / \mathrm{s},-1.75 \mathrm{~m} / \mathrm{s}$ and $-1.85 \mathrm{~m} / \mathrm{s}$ for the 50,100 , and 200 wood pellets cases, respectively.

\section{Hosted file}

image17.emf available at https://authorea.com/users/430819/articles/534349-measuring-binaryfluidization-of-non-spherical-and-spherical-particles-using-machine-learning-aidedimage-processing

Figure 17. The distribution of the (a) horizontal and (b) vertical velocity of wood particles with varying bed height and superficial gas velocity all with 100 wood particles.

\section{Hosted file}

image18.emf available at https://authorea.com/users/430819/articles/534349-measuring-binaryfluidization-of-non-spherical-and-spherical-particles-using-machine-learning-aidedimage-processing

Figure 18. The distribution of the (a) horizontal and (b) vertical velocity of wood particles at $3 \mathrm{U}_{\mathrm{mf}}$, $\mathrm{H}_{\mathrm{b} 0}=15.24 \mathrm{~cm}$, and varying wood particle counts.

\section{Conclusion}

Using high-speed imaging, the current study provides detailed experimental data on the effects of nonspherical wood particle counts, static bed height, and superficial gas velocity on the binary fluidization behavior of a Geldart-D particle type system. Machine learning-aided image analysis is applied to extract particles mask of a specific type followed by PIV and PTV analysis to quantify the LDPE particle velocity field and the velocity and orientation of wood particles, respectively.

In general, the fluidization for LDPE particles only and LDPE/wood particles behave rather differently. While LDPE particles slugging are achieved at a low gas flow rate of $1.4 \mathrm{U}_{\mathrm{mf}}$ and $2 \mathrm{U}_{\mathrm{mf}}$, the bed transitions to a spouting regime at higher flow rates of $3 \mathrm{U}_{\mathrm{mf}}$ and $3.4 \mathrm{U}_{\mathrm{mf}}$. This transition is clearly observed in the mean and standard deviation of the expanded bed height. To our knowledge, the spouting behavior of Geldart D type particles has not been reported before under uniform flow conditions using porous distributor plates 
without gas jets. The resultant maximum downward velocity within the fountain regions is significantly lower than those obtained using a central gas jet even with lower gas velocity.

With the addition of wood particles, the spouting phenomenon has not been observed under all tested conditions varying static bed height, wood particles counts, and fluidization velocity. At a low gas flow rate $\left(1.5 \mathrm{U}_{\mathrm{mf}}\right)$, the wood and LDPE particles remain perfectly segregated under different static bed heights and wood particle counts while the LDPE particles form slugging flow. At $2.0 \mathrm{U}_{\mathrm{mf}}-3.7 \mathrm{U}_{\mathrm{mf}}$, wood and LDPE particles are mixed and binary slugging flows are formed. At $2 \mathrm{U}_{\mathrm{mf}}$, adding wood particles also causes a $26 \%$ increase in the rising speed of the slug and a $112 \%$ increase of the maximum fall speed after the slugs disintegration. Furthermore, the spatially averaged vertical velocity shows quasi-periodic fluctuations for all cases except for the spouting bed for the LDPE only case at $3 \mathrm{U}_{\mathrm{mf}}$. At $2 \mathrm{U}_{\mathrm{mf}}$, the mean amplitude of the signal almost doubled, while the mean frequency sees a $38 \%$ increase with the addition of wood particles. The increase of fluidization velocity for the binary fluidization case to $3 \mathrm{U}_{\mathrm{mf}}$ causes a $110 \%$ increase of the mean amplitude and a 35\% decrease in mean frequency. Furthermore, the mean bed height seems to collapse at different static bed heights after nondimenlization. The evolution of the spatially averaged vertical velocities behaves similarly under different static bed heights. After using the characteristics falling velocity and time for nondimenlization, the mean periods seem to collapse reasonably well between different bed height and fluidization velocities. However, the scaling only works for mean amplitude under the same static bed height.

Finally, the wood particles preferentially orientate themselves towards $0^{\circ}$ (vertical) and $90^{\circ}$ (horizontal) for all tested cases at dilute and dense region of the bed respectively. No significant orientation distribution differences are identified under tested conditions. The horizontal wood particle velocities show a Gaussian distribution for all cases and a varying degree of bimodal distribution for the vertical velocities. One unresolved issue is the systematic quantification of distribution. As the result shows, the wood particles demonstrate varying degrees of segregation with varying superficial gas velocity and wood particle fraction. The current system has limited wood particle counts which inherently causes the segregation analysis noisy. A larger system with a significant number of particles is preferred for meaningful segregation analysis and is deferred to future studies.

\section{Acknowledgement}

This work was performed in support of the US Department of Energy's EERE Bioenergy Technologies Office (BETO) as part of the BETO Consortium for Computational Physics and Chemistry (CCPC). The Research was executed through the NETL Research and Innovation Center's BETO CCPC. This research was also supported in part by an appointment to the National Energy Technology Laboratory Research Participation Program, sponsored by DOE and administered by the Oak Ridge Institute for Science and Education. Research performed by Leidos Research Support Team staff was conducted under the RSS contract $89243318 \mathrm{CFE} 000003$

\section{Disclaimer}

This paper was prepared as an account of work sponsored by an agency of the United States Government. Neither the United States Government nor any agency thereof, nor any of their employees, makes any warranty, express or implied, or assumes any legal liability or responsibility for the accuracy, completeness, or usefulness of any information, apparatus, product, or process disclosed, or represents that its use would not infringe privately owned rights. Reference herein to any specific commercial product, process, or service by trade name, trademark, manufacturer, or otherwise does not necessarily constitute or imply its endorsement, recommendation, or favoring by the United States Government or any agency thereof. The views and opinions of authors expressed herein do not necessarily state or reflect those of the United States Government or any agency thereof. 


\section{References}

(1) Cui, H.; Grace, J. R. Fluidization of Biomass Particles: A Review of Experimental Multiphase Flow Aspects. Chem. Eng. Sci.2007, 62 (1-2), 45-55.

(2) Pinto, F.; Franco, C.; André, R. N.; Tavares, C.; Dias, M.; Gulyurtlu, I.; Cabrita, I. Effect of Experimental Conditions on Co-Gasification of Coal, Biomass and Plastics Wastes with Air/Steam Mixtures in a Fluidized Bed System. In Fuel ; Elsevier, 2003; Vol. 82, pp 1967-1976. https://doi.org/10.1016/S0016-2361(03)00160-1.

(3) Xue, Y.; Zhou, S.; Brown, R. C.; Kelkar, A.; Bai, X. Fast Pyrolysis of Biomass and Waste Plastic in a Fluidized Bed Reactor. Fuel2015 , 156 (September), 40-46. https://doi.org/10.1016/j.fuel.2015.04.033.

(4) Gao, X.; Yu, J.; Lu, L.; Li, C.; Rogers, W. A. Development and Validation of SuperDEM-CFD Coupled Model for Simulating Non-Spherical Particles Hydrodynamics in Fluidized Beds. Chem. Eng. J.2021 , 420 , 127654. https://doi.org/10.1016/j.cej.2020.127654.

(5) Daghooghi, M.; Borazjani, I. The Effects of Irregular Shape on the Particle Stress of Dilute Suspensions. J. Fluid Mech.2018 , 839 , 663-692. https://doi.org/10.1017/jfm.2018.65.

(6) Clift, R., Grace, J.R., Weber, M. E. . Bubbles, Drops and Particles ; Academic Press, 1978. https://doi.org/10.1017/S0022112079221290.

(7) Gao, X.; Yu, J.; Li, C.; Panday, R.; Xu, Y.; Li, T.; Ashfaq, H.; Hughes, B.; Rogers, W. A. Comprehensive Experimental Investigation on Biomass-Glass Beads Binary Fluidization: A Data Set for CFD Model Validation. AIChE J. 2020 , 66 (2), 1-18. https://doi.org/10.1002/aic.16843.

(8) Lu, L.; Gao, X.; Shahnam, M.; Rogers, W. A. Coarse Grained Computational Fluid Dynamic Simulation of Sands and Biomass Fluidization with a Hybrid Drag. AIChE J. 2020 , 66 (4), 1-10. https://doi.org/10.1002/aic.16867.

(9) Zhang, Y.; Jin, B.; Zhong, W.; Ren, B.; Xiao, R. Characterization of Fluidization and Segregation of Biomass Particles by Combining Image Processing and Pressure Fluctuations Analysis. Int. J. Chem. React. Eng. 2009 , 7 . https://doi.org/10.2202/1542-6580.2045.

(10) Lu, L.; Yu, J.; Gao, X.; Xu, Y.; Shahnam, M.; Rogers, W. A. Experimental and Numerical Investigation of Sands and Geldart A Biomass Co-Fluidization. AIChE J. 2020, 66 (6). https://doi.org/10.1002/aic.16969.

(11) Buist, K. A.; Jayaprakash, P.; Kuipers, J. A. M.; Deen, N. G.; Padding, J. T. Magnetic Particle Tracking for Nonspherical Particles in a Cylindrical Fluidized Bed. AIChE J. 2017 , 63(12), 5335-5342. https://doi.org/10.1002/aic.15854.

(12) Fotovat, F.; Ansart, R.; Hemati, M.; Simonin, O.; Chaouki, J. Sand-Assisted Fluidization of Large Cylindrical and Spherical Biomass Particles: Experiments and Simulation. Chem. Eng. Sci.2015 , 126 , 543-559. https://doi.org/10.1016/j.ces.2014.12.022.

(13) Chen, X.; Zhong, W.; Heindel, T. J. Using Stereo XPTV to Determine Cylindrical Particle Distribution and Velocity in a Binary Fluidized Bed. AIChE J. 2019, 65 (2), 520-535. https://doi.org/10.1002/aic.16485.

(14) Vollmari, K.; Jasevičius, R.; Kruggel-Emden, H. Experimental and Numerical Study of Fluidization and Pressure Drop of Spherical and Non-Spherical Particles in a Model Scale Fluidized Bed. Powder Technol. 2016 , 291 , 506-521. https://doi.org/10.1016/j.powtec.2015.11.045.

(15) Pal, N. R.; Pal, S. K. A Review on Image Segmentation Techniques.Pattern Recognit. 1993 , 26 (9), 1277-1294. https://doi.org/10.1016/0031-3203(93)90135-J.

(16) Yin, S.; Zhong, W.; Song, T.; Lu, P.; Chen, Y. Clusters Identification and Meso-Scale Structures in a Circulating Fluidized Bed Based on Image Processing. Adv. Powder Technol. 2019 ,30 (12), 3010-3020. https://doi.org/10.1016/j.apt.2019.09.008. 
(17) Jiang, Z.; Hagemeier, T.; Bück, A.; Tsotsas, E. Experimental Measurements of Particle Collision Dynamics in a Pseudo-2D Gas-Solid Fluidized Bed. Chem. Eng. Sci. 2017 , 167, 297-316. https://doi.org/10.1016/j.ces.2017.04.024.

(18) Yevick, A.; Hannel, M.; Grier, D. G. Machine-Learning Approach to Holographic Particle Characterization. Opt. Express2014, 22 (22), 26884. https://doi.org/10.1364/OE.22.026884.

(19) Rodellar, J.; Alférez, S.; Acevedo, A.; Molina, A.; Merino, A. Image Processing and Machine Learning in the Morphological Analysis of Blood Cells. Int. J. Lab. Hematol. 2018 , 40(February), 46-53. https://doi.org/10.1111/ijlh.12818.

(20) Lai, Z.; Chen, Q. Reconstructing Granular Particles from X-Ray Computed Tomography Using the TWS Machine Learning Tool and the Level Set Method. Acta Geotech. 2019 , 14 (1), 1-18. https://doi.org/10.1007/s11440-018-0759-x.

(21) Guo, Q.; Ye, M.; Yang, W.; Liu, Z. A Machine Learning Approach for Electrical Capacitance Tomography Measurement of Gas-Solid Fluidized Beds. AIChE J. 2019 , 65 (6). https://doi.org/10.1002/aic.16583.

(22) McCoy, J. T.; Auret, L. Machine Learning Applications in Minerals Processing: A Review. Miner. Eng. 2019 , 132, 95-109. https://doi.org/10.1016/j.mineng.2018.12.004.

(23) Hussain, R.; Alican Noyan, M.; Woyessa, G.; Retamal Marín, R. R.; Antonio Martinez, P.; Mahdi, F. M.; Finazzi, V.; Hazlehurst, T. A.; Hunter, T. N.; Coll, T.; Stintz, M.; Muller, F.; Chalkias, G.; Pruneri, V. An Ultra-Compact Particle Size Analyser Using a CMOS Image Sensor and Machine Learning. Light Sci. Appl. 2020 , 9 (1). https://doi.org/10.1038/s41377-020-0255-6.

(24) Shao, S.; Mallery, K.; Hong, J. Machine Learning Holography for Measuring 3D Particle Distribution. Chem. Eng. Sci.2020, 225, 115830. https://doi.org/10.1016/j.ces.2020.115830.

(25) Li, C.; Gao, X.; Rowan, S.; Hughes, B.; Harris, J.; Rogers, W.Experimental Investigation on the Binary/Ternary Fluidization Behavior of Geldart D Type Spherical LDPE, Geldart D Type Cylindrical Wood and Geldart B Type Sand Particles ; 2021. https://doi.org/10.2172/1776642.

(26) Tucker, J. R.; Shadle, L. J.; Benyahia, S.; Koepke, M. E.; Mei, J.; Guenther, C. Improvement in Precision, Accuracy, and Efficiency in Standardizing the Characterization of Granular Materials. InProceedings of the ASME 2013 International Mechanical Engineering Congress and Exposition ; 2013; pp 1-9.

(27) Epstein, N.; Grace, J. R. Spouted and Spout-Fluid Beds: Fundamentals and Applications ; Epstein, N., Grace, J. R., Eds.; Cambridge University Press: Cambridge, 2010; Vol. 9780521517. https://doi.org/10.1017/CBO9780511777936.

(28) Otsu, N. A Threshold Selection Method from Gray-Level Histograms.IEEE Trans. Syst. Man. Cybern. 1979 , 9 (1), 62-66. https://doi.org/10.1109/TSMC.1979.4310076.

(29) Arganda-Carreras, I.; Kaynig, V.; Rueden, C.; Eliceiri, K. W.; Schindelin, J.; Cardona, A.; Seung, H. S. Trainable Weka Segmentation: A Machine Learning Tool for Microscopy Pixel Classification.Bioinformatics 2017 , 33 (15), 2424-2426. https://doi.org/10.1093/bioinformatics/btx180.

(30) Ouellette, N. T.; Xu, H.; Bodenschatz, E. A Quantitative Study of Three-Dimensional Lagrangian Particle Tracking Algorithms. Exp. Fluids 2006 , 40 (2), 301-313. https://doi.org/10.1007/s00348-0050068-7.

(31) Thielicke, W.; Stamhuis, E. J. PIVlab-Time-Resolved Digital Particle Image Velocimetry Tool for MATLAB. Publ. under BSD Licens. Program. with MATLAB 2014, 7 (0.246), R14.

(32) Mathur, K. B.; Gishler, P. E. A Technique for Contacting Gases with Coarse Solid Particles. AIChE J. 1955 , 1 (2), 157-164. https://doi.org/10.1002/aic.690010205. 
(33) Sutanto, W.; Epstein, N.; Grace, J. R. Hydrodynamics of Spout-Fluid Beds. Powder Technol. 1985 , 44 (3), 205-212. https://doi.org/10.1016/0032-5910(85)85001-4.

(34) He, Y.-L.; Lim, C. J.; Grace, J. R. Spouted Bed and Spout-Fluid Bed Behaviour in a Column of Diameter 0.91 M. Can. J. Chem. Eng.1992, 70 (5), 848-857. https://doi.org/10.1002/cjce.5450700505.

(35) Stewart, P. S. B.; Davidson, J. F. Slug Flow in Fluidised Beds.Powder Technol. 1967 , 1 (2), 61-80. https://doi.org/10.1016/0032-5910(67)80014-7.

(36) He, Y.-L.; Qin, S.-Z.; Lim, C. J.; Grace, J. R. Particle Velocity Profiles and Solid Flow Patterns in Spouted Beds. Can. J. Chem. Eng. 1994 , 72 (4), 561-568. https://doi.org/10.1002/cjce.5450720402.

(37) Olazar, M.; San José, M. J.; Izquierdo, M. A.; de Salazar, A. O.; Bilbao, J. Effect of Operating Conditions on Solid Velocity in the Spout, Annulus and Fountain of Spouted Beds. Chem. Eng. Sci.2001 , 56 (11), 3585-3594. https://doi.org/10.1016/S0009-2509(01)00022-7.

(38) Karlsson, S.; Niklasson Björn, I.; Folestad, S.; Rasmuson, A. Measurement of the Particle Movement in the Fountain Region of a Wurster Type Bed. Powder Technol. 2006 , 165 (1), 22-29. https://doi.org/10.1016/j.powtec.2006.03.014. 